\title{
Accuracy Analysis of the Sine-wave Parameters Estimation by Means of the Windowed Three-Parameter Sine-Fit Algorithm
}

\author{
Daniel Belega ${ }^{1}$ and Dario Petri ${ }^{2}$ \\ ${ }^{1}$ Department of Measurements and Optical Electronics, Politehnica University Timişoara, \\ Bv. V. Pârvan, Nr. 2, 300223, Timişoara, Romania, \\ Phone: +40 2564033 65, Fax : +40 2564033 62, E-mail: daniel.belega@upt.ro \\ ${ }^{2}$ Department of Electrical Engineering, University of Trento, \\ Trento 38123, Italy, \\ Phone: +39 0461 883902, Fax: +39 0461 882093, E-mail: dario.petri@unitn.it
}

\begin{abstract}
This paper investigates the accuracy of the sine-wave parameter estimators provided by the Weighted Three-Parameter Sine-Fit (W3PSF) algorithm when a generic cosine window is adopted. Simple expressions for the estimators are derived, which allows a very simple implementation of that algorithm. Moreover, it is shown that the W3PSF algorithm can be well approximated by the classical weighted Discrete Time Fourier Transform (DTFT) when the number of analysed waveform cycles is high enough. Under that constraint the asymptotic mean square errors (MSEs) of the estimated parameters and the expected sum-squared fitting error can also be evaluated with good accuracy using very simple expressions. Then, the statistical performances of the W3PSF algorithm and the classical 3PSF algorithm are compared through computer simulations in the case of noisy or noisy and harmonically distorted sinewaves. The performed analysis allows us to identify when the W3PSF algorithm outperforms the classical 3PSF algorithm.
\end{abstract}

Keywords: Non-coherent sampling, parameter estimation, statistical analysis, three-parameter sine-fit algorithm, windowing.

\section{Introduction}

Many engineering applications require accurate estimates of the parameters of noisy sine-waves. To this aim sine-fit algorithms based on the least squares approach are often adopted [1-15]. Indeed, these algorithms are quite simple to implement and return asymptotically efficient estimates when the analysed sine-wave is affected by additive white Gaussian noise [1]. Two different sine-fit algorithms are available 
in the literature, that is the three-parameter sine-fit (3PSF) algorithm and the four-parameter sine-fit (4PSF) algorithm [3-6], according to the number of sine-wave parameters to be estimated. Both algorithms are recommended in the IEEE Standards for the dynamic characterization of digitizing waveform recorders [3] or analog-to-digital converters [4]. The 3PSF algorithm assumes that the sinewave frequency is a-priori known, and the remaining waveform parameters are estimated through a linear least-square approach. Conversely, the 4PSF algorithm estimates also the sine-wave frequency, thus resulting in a non-linear least-square problem, whose solution is achieved iteratively. When the sine-wave frequency is known with uncertainty, a criterion for the selection of the most accurate algorithm has been derived in [7] on the basis of the parsimony principle [2]. The analytical expressions for the sine-wave parameter estimators provided by the 3PSF algorithm are given in [5]. Unfortunately, they are quite complicated unlike other approaches, such as those based on the Discrete Fourier Transform (DFT) [1631]. In practice, sine-waves are often affected by narrow-band disturbances, such as harmonics or inter-harmonics. To reduce the detrimental effect on these disturbances the analysed waveform is often weighted by means of a suitable window function, in both non-parametric and parametric methods [1, 2, 16-18, 20, 22, 27-29]. Unfortunately, to the best of the authors' knowledge, the accuracy of the weighted sine-fit algorithms has not yet been analysed in the scientific literature. This is the aim of this paper. At first closed form expressions for the sine-wave parameter estimators returned by the Weighted 3PSF (W3PSF) algorithm are derived assuming that a generic $H$-term cosine window $(H \geq 1)$ is adopted. The obtained expressions are simple linear combinations of the values of the weighted Discrete Time Fourier Transform (DTFT) evaluated at DC and at the sine-wave frequency. They allow us to analyse the effect of both narrow-band disturbances and wideband noise on the accuracy of the estimated sine-wave parameters. In particular, it is shown that the W3PSF algorithm and the classical weighted DTFT return almost the same estimates when quasi-coherent sampling occurs or when the number of analysed sinewave cycles is high enough. As a consequence, the expressions for the asymptotic (high number of samples) Mean Square Errors (MSEs) of the parameter estimates returned by the W3PSF algorithm and the related expected sum-squared fitting error [8] are easily achieved.

The paper is organized as follows. In Section 2 the expressions for the sine-wave parameter estimators returned by the W3PSF algorithm are derived and some remarks about the effect of narrow-band disturbances on the parameter estimation accuracy are drawn. Moreover, simple expressions for the asymptotic MSEs of the estimated parameters and the expected sum-squared fitting error provided by the W3PSF algorithm when the number of sine-wave cycles is high enough are provided in Section 3. In Section 4 the MSEs of the estimated parameters and the expected sum-squared fitting error of the W3PSF algorithm and the classical 3PSF algorithm are compared to each other in the case of noisy or noisy and harmonically distorted sine-waves. As a result, conditions under which the W3PSF algorithm outperforms 
the classical 3PSF algorithm are identified. Also, the MSEs returned by simulations are compared with the derived expressions. Finally, Section 5 concludes the paper.

\section{The W3PSF algorithm}

The analyzed discrete-time noisy sine-wave is modelled as:

$$
x(m)=A \sin \left(2 \pi f\left(m-\frac{M-1}{2}\right)+\phi\right)+d+e(m)=s(m)+e(m), \quad m=0,1,2, \ldots, M-1
$$

where $s(\cdot)$ is the discrete-time sine-wave of amplitude $A$, frequency $f$, and phase $\phi, d$ is the offset and $e(\cdot)$ is an additive white Gaussian noise with zero mean and variance $\sigma^{2}$. Without loss of generality an odd number of acquired samples $M$ is assumed, so that the time reference can be set exactly in the center of the observation interval, thus simplifying the derived expressions (as it will be shown in the following).

The sine-wave frequency $f$ is chosen in the range $(0,0.5)$ to satisfy the Nyquist theorem. It can be expressed as:

$$
f=\frac{f_{\text {in }}}{f_{s}}=\frac{v}{M}=\frac{l+\delta}{M}
$$

where $f_{\text {in }}$ is the frequency of the acquired continuous-time sine-wave, $f_{s}$ is the sampling frequency (both expressed in $\mathrm{Hz}$ ), $v$ is the number of acquired signal cycles or the sine-wave frequency expressed in bins, $l$ is the rounded value of $v$, and $\delta=v-l$, with $\delta \in[-0.5,0.5)$. In particular, $\delta=0$ when coherent sampling occurs.

The sine-wave $s(\cdot)$ can be also re-written as:

$$
s(m)=\frac{1}{2}\left(p e^{j 2 \pi f\left(m-\frac{M-1}{2}\right)}+p^{*} e^{-j 2 \pi f\left(m-\frac{M-1}{2}\right)}\right), \quad m=0,1,2, \ldots
$$

where $\stackrel{\Delta}{=} A e^{j \phi}$.

It is well-known that the influence on the estimated sine-wave parameters of narrow-band disturbances can be reduced by weighting the analysed signal $x(\cdot)$ by a suitable window function. In this paper the cosine class windows $(H \geq 1)$ are considered [32, 33], that is:

$$
w(m)=\sum_{h=0}^{H-1} c_{h} \cos \left(2 \pi \frac{h}{M}\left(m-\frac{M-1}{2}\right)\right), \quad m=0,1, \ldots, M-1
$$

where $c_{h}, h=0, \ldots H$ - 1 are the window coefficients and $H \geq 1$ is the number of window terms. Note that the particular case $H=1$ corresponds to the rectangular window, i.e. to the absence of the weighting.

The three-parameter sine-fit approach assumes that an estimate $\hat{v}$ of the number of acquired sinewave cycles is known a-priori [3-7] The following theorem holds. 


\section{Theorem 1}

The estimators for the sine-wave complex-amplitude $p$ and the offset $d$ returned by the W3PSF algorithm are:

$$
\hat{p}=\frac{2}{\widetilde{W}(0)}\left(\alpha_{11} X_{\tilde{w}}(\hat{v})+\alpha_{12} X_{\tilde{w}}^{*}(\hat{v})+\alpha_{13} X_{\widetilde{w}}(0)\right)
$$

and

$$
\hat{d}=\frac{1}{\tilde{W}(0)}\left(\alpha_{13} X_{\tilde{w}}(\hat{v})+\alpha_{13} X_{\tilde{w}}^{*}(\hat{v})+\alpha_{33} X_{\tilde{w}}(0)\right)
$$

where $X_{\tilde{w}}(\cdot)$ is the Discrete Time Fourier Transform (DTFT) of the analysed signal weighted by the squared window $w^{2}(m)=\tilde{w}(m), \tilde{W}(\cdot)$ is the DTFT of the squared window $\tilde{w}(\cdot)$, and

$$
\alpha_{11} \stackrel{\Delta}{=} \frac{1-a^{2}}{1-2 a^{2}-b^{2}+2 a^{2} b}, \alpha_{12} \stackrel{\Delta}{=} \frac{a^{2}-b}{1-2 a^{2}-b^{2}+2 a^{2} b}, \alpha_{13} \stackrel{\Delta}{=}-\frac{a}{1+b-2 a^{2}}, \alpha_{33} \stackrel{\Delta}{=} \frac{1+b}{1+b-2 a^{2}},
$$

in which

$$
a=\frac{\Delta \tilde{W}(-\hat{v})}{\tilde{W}(0)} \text { and } b=\frac{\Delta \tilde{W}(-2 \hat{v})}{\tilde{W}(0)} .
$$

The proof of that theorem is given in Appendix A.

Since $p=A e^{j \phi}$, the expressions for the sine-wave amplitude and phase estimators can be easily derived from (5):

$$
\hat{A}=|\hat{p}|=\frac{2}{\widetilde{W}(0)}\left|\alpha_{11} X_{\widetilde{w}}(\hat{v})+\alpha_{12} X_{\widetilde{w}}^{*}(\hat{v})+\alpha_{13} X_{\widetilde{w}}(0)\right|
$$

and

$$
\hat{\phi}=\operatorname{angle}\{\hat{p}\}=\operatorname{angle}\left\{\alpha_{11} X_{\widetilde{w}}(\hat{v})+\alpha_{12} X_{\widetilde{w}}^{*}(\hat{v})+\alpha_{13} X_{\widetilde{w}}(0)\right\} .
$$

It is worth noticing that the expressions (9), (10), and (6), for the estimators $\hat{A}, \hat{\phi}$, and $\hat{d}$ are explicitly related to the DTFT of the analyzed waveform weighted by the squared window $\tilde{w}(\cdot)$. Moreover, they are simpler than those published in the literature in the particular case of the rectangular window [5]. In addition, these expressions do not require the calculation of the pseudoinverse matrix, as occurs when using the classical least-squares approach.

Using mathematical induction it is easy to show the following

\section{Proposition}

The squared window of a $H$-term cosine window $(H \geq 2)$ with coefficients $c_{h}, h=0,1, \ldots, H-1$ is a $(2 H-1)$ term cosine window with coefficients: 


$$
\begin{gathered}
\widetilde{c}_{0}=c_{0}^{2}+0.5 \sum_{h=1}^{H-1} c_{h}^{2}=N N P G_{w}, \\
\widetilde{c}_{h}=2 \sum_{n=-(H-1)}^{H-1-h} q_{|n|} q_{|n+h|}, \quad h=1,2, \ldots, 2 H-2
\end{gathered}
$$

in which $q_{k}=\left\{\begin{array}{ll}c_{0}, & k=0 \\ 0.5 c_{k}, & k=1,2, \ldots H-1\end{array}\right.$, while $N N P G_{w}$ is the Normalized Noise Power Gain parameter of the original window.

Other window figures of merit that will be used in the following, are the Normalized Peak Signal Gain (NPSG) and the Equivalent Noise BandWidth (ENBW). For the squared window $\tilde{w}(\cdot)$ the parameters NPSG, NNPG, and ENBW are expressed as follows [32]:

$$
\begin{gathered}
N P S G_{\tilde{w}} \stackrel{\Delta}{=} \frac{1}{M} \sum_{m=0}^{M-1} \tilde{w}(m)=\widetilde{c}_{0}=N N P G_{w}, \\
N N P G_{\tilde{w}} \stackrel{\Delta}{=} \frac{1}{M} \sum_{m=0}^{M-1} \tilde{w}^{2}(m)=\widetilde{c}_{0}^{2}+0.5 \sum_{h=1}^{H-1} \tilde{c}_{h}^{2},
\end{gathered}
$$

and [32]:

$$
\operatorname{ENBW}_{\widetilde{w}}=\frac{\Delta N P G_{\widetilde{w}}}{\left(N P S G_{\tilde{w}}\right)^{2}}=1+0.5 \sum_{h=1}^{H-1}\left(\frac{\widetilde{c}_{h}}{\widetilde{c}_{0}}\right)^{2} .
$$

It is worth to observe that $\tilde{W}(\hat{v}) \cong 0$ and $\tilde{W}(2 \hat{v}) \cong 0$ when a suitable window is used, a sufficiently large number of samples $M$ is acquired and enough sine-wave cycles $v$ are observed (i.e. $M>>v>>1$ ). In that case (8) returns $a \cong 0$ and $b \cong 0$, which replaced in (7) leads to $\alpha_{11} \cong \alpha_{33} \cong 1$, and $\alpha_{12} \cong \alpha_{13} \cong 0$. Thus, (9), (10), and (6) provide:

$$
\begin{gathered}
\hat{A} \cong \frac{2\left|X_{\tilde{w}}(\hat{v})\right|}{\tilde{W}(0)}, \\
\hat{\phi} \cong \operatorname{angle}\left\{X_{\tilde{w}}(\hat{v})\right\},
\end{gathered}
$$

and

$$
\hat{d} \cong \frac{X_{\tilde{w}}(0)}{\tilde{W}(0)}
$$

respectively, which coincide with the expressions used to the classical weighted DTFT algorithm.

In fact, under the assumed constraints the spectral interference from the sine-wave image component become negligible, so that the W3PSF and the classical weighted DTFT algorithms return very close 
parameter estimates. It is worth noticing that the expressions (15)-(17) hold also in coherent or quasicoherent (i.e when $\delta \cong 0$ ) sampling mode since in these modes we have $\hat{v} \cong l$.

It is worth observing that $\tilde{W}(v)$ and $\tilde{W}(2 v)$ assume significant values when $v$ is small. Thus (5)-(8) show that the sine-wave parameter estimators returned by the W3PSF algorithm are heavily affected by the term $X_{\tilde{w}}^{*}(\hat{v})$, which is related to the image component. Moreover, its contribution increases as the error $\Delta v=\hat{v}-v$ of the a-priori estimate of the sine-wave frequency increases, as shown by (A.12). This effect is more evident when the rectangular window is used due to its high spectrum sidelobe level.

\section{Accuracy of the W3PSF algorithm}

In this Section the MSE of the sine-wave parameter estimators returned by the W3PSF algorithm and the related sum-squared fitting error are evaluated under the assumption that (15)-(17) hold, which occurs in the case of coherent or quasi-coherent sampling or when the number of observed sine-wave cycles $v$ is high enough. In particular, the MSEs of the estimators returned by the W3PSF algorithm are expressed by the following

\section{Theorem 2}

The asymptotic MSEs for the estimators $\hat{A}, \hat{\phi}$, and $\hat{d}$ returned by the W3PSF algorithm when the number of observed sine-wave cycles is high can be approximated by:

$$
\begin{gathered}
\operatorname{MSE}[\hat{A}]=\operatorname{bias}^{2}[\hat{A}]+\sigma_{\hat{A}}^{2} \cong A^{2}\left(\frac{\pi^{2}}{6}+\sum_{h=1}^{2 H-2} \frac{(-1)^{h}}{h^{2}} \frac{\widetilde{c}_{h}}{\widetilde{c}_{0}}\right)^{2} \Delta^{4} v+\frac{2}{M} \operatorname{ENBW}_{\widetilde{w}} \sigma^{2}, \\
M S E[\hat{\phi}] \cong \sigma_{\hat{\phi}}^{2} \cong \frac{1}{M \cdot S N R} E^{2}{ }^{2} W_{\tilde{w}},
\end{gathered}
$$

and

$$
\operatorname{MSE}[\hat{d}] \cong \sigma_{\hat{d}}^{2} \cong \frac{1}{M} E N B W_{\widetilde{w}} \sigma^{2}
$$

where $\Delta v=\hat{v}-v$ and $S N R=A^{2} /\left(2 \sigma^{2}\right)$ is the Signal-to-Noise Ratio.

The proof of that theorem is given in Appendix B.

From (18)-(20) it follows that $\operatorname{MSE}[\hat{\phi}]$ and $M S E[\hat{d}]$ do not depend on $\Delta v[1]$, unlike $M S E[\hat{A}]$. The same holds for the sine-wave phase, even though it can be shown that the independence of $v$ occurs because the time reference has been set exactly in the center of the observation interval. 
The obtained MSEs are compared with the lower bound MSEs for unbiased estimators, which corresponds to the asymptotic Cramér-Rao Lower Bounds (CRLB) [1]:

$$
\begin{gathered}
(\operatorname{MSE}[\hat{A}])_{C R}=\left(\sigma_{\hat{A}}^{2}\right)_{C R} \cong \frac{2}{M} \sigma^{2}, \\
(\operatorname{MSE}[\hat{\phi}])_{C R}=\left(\sigma_{\hat{\phi}}^{2}\right)_{C R} \cong \frac{1}{M \cdot S N R},
\end{gathered}
$$

and

$$
(\operatorname{MSE}[\hat{d}])_{C R}=\left(\sigma_{\hat{d}}^{2}\right)_{C R} \cong \frac{1}{M} \sigma^{2} .
$$

Expression (18) confirms that, when a high accuracy frequency estimate is available (i.e. $\Delta \hat{v} \cong 0$ ), the bias is almost null and so the statistical efficiency of the amplitude estimator (i.e. the ratio between the CRLB and the $\operatorname{MSE}[\hat{A}]$ ) is almost equal to $1 / E N B W_{\widetilde{w}}$. Similarly, expressions (19) and (22), and (20) and (23) show that the statistical efficiencies of the phase and offset estimators are almost equal to $1 / E N B W_{\tilde{w}}$.

The fitting error is defined as [8]:

$$
\varepsilon(m) \stackrel{\Delta}{=} \hat{s}(m)-s(m), \quad m=0,1,2, \ldots, M-1
$$

where $\hat{s}(\cdot)$ is the fitting sine-wave given by:

$$
\hat{s}(m)=A \sin \left(2 \pi \frac{\hat{v}}{M}\left(m-\frac{M-1}{2}\right)+\hat{\phi}\right)+\hat{d}, \quad m=0,1,2, \ldots, M-1
$$

in which $\hat{A}, \hat{\phi}$, and $\hat{d}$ are the estimated amplitude, phase, and offset parameters.

The sum-squared fitting error is defined as [8]:

$$
\gamma \stackrel{\Delta}{=} \frac{1}{M} \sum_{m=0}^{M-1} \varepsilon^{2}(m) .
$$

and its expected value is given by the following

\section{Theorem 3}

The asymptotic expected sum-squared fitting error obtainable by the W3PSF algorithm when the number of observed sine-wave cycles is high can be approximated by:

$$
E[\gamma] \cong \frac{3 E N B W_{\tilde{w}}}{M} \sigma^{2}+\frac{\pi^{2} A^{2}}{6} \operatorname{MSE}(\hat{v}) .
$$

The proof of that theorem is given in Appendix C. 
In particular, when the rectangular window is adopted $\left(E N B W_{\tilde{w}}=1\right)$, (27) coincides with the related expression derived in [8], that is:

$$
E[\gamma] \cong \frac{3}{M} \sigma^{2}+\frac{\pi^{2} A^{2}}{6} \operatorname{MSE}(\hat{v}) .
$$

As simulations show, (27) holds with good accuracy also in the case of harmonically distorted sine-waves since the contribution of harmonics to the sine-wave parameter estimates is negligible when windowing is applied and a suitably high number of sine-wave cycles are observed.

Observe also that (18), (19), (20), and (27) show that the reduction of the influence of narrow-band disturbances on the estimation accuracy provided by windowing, increases the estimator sensitivity to wideband noise by a factor which is related to the ENBW of the square of the adopted window.

\section{Computer simulations}

The aim of this Section is to verify through computer simulations the accuracies of the expressions derived in the previous two Sections and to compare the performance of the W3PSF and the 3PSF algorithms when noisy or noisy and harmonically distorted sine-waves are analysed. Sine-waves used in the simulations have amplitude $A=1$ and offset $d=0.1$.

Firstly the results returned by the 3PSF and W3PSF algorithm implemented using both the classical least squares procedure and the proposed relationships are compared to each other. Fig. 1 shows the expected sum-squared fitting error $\mathrm{E}[\gamma]$ obtained by the considered algorithms as a function of $v$ when the rectangular window or the two-term Maximum Sidelobe Decay (MSD) window (known also as the Hann window) [33] are used and $M=17$ and 129, respectively. The waveform SNR is $40 \mathrm{~dB}$ and $\Delta v=7 \cdot 10^{-3}$ cycles. In this case the second term in (27) is higher than the first one so that the effect of frequency uncertainty prevails on wideband noise. The frequency $v$ was varied in the range $[1.01,6)$ with a step of 0.1 . For each considered value of $v, 10000$ values of the phase $\phi$ were chosen at random in the range $[0,2 \pi) \mathrm{rad}$. The theoretical values returned by (27) and (28) for the rectangular and the two-term MSD windows, respectively, are also reported in the figure. 


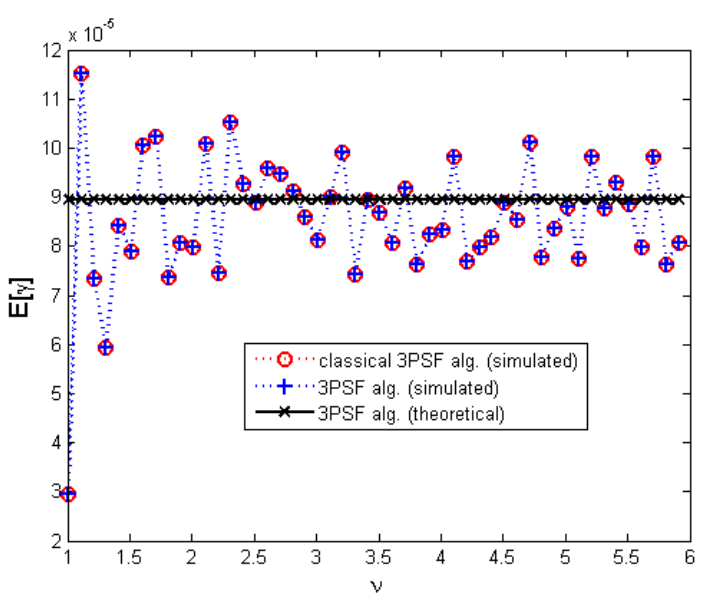

(a)

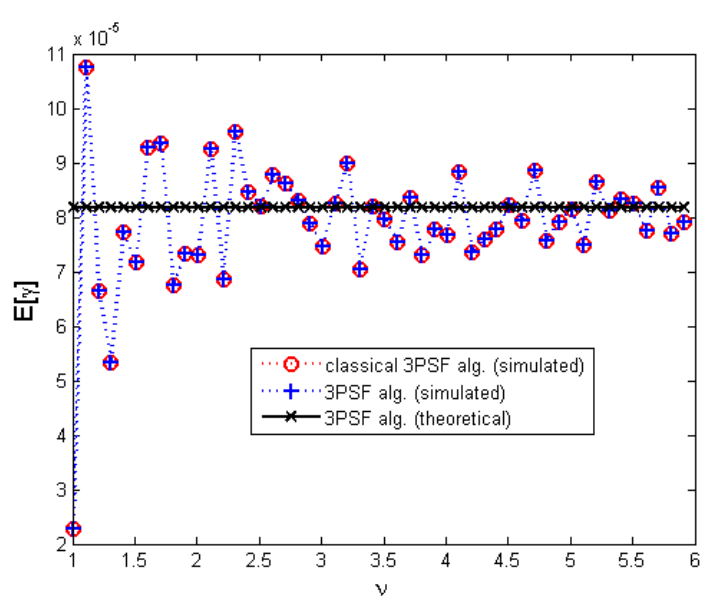

(c)

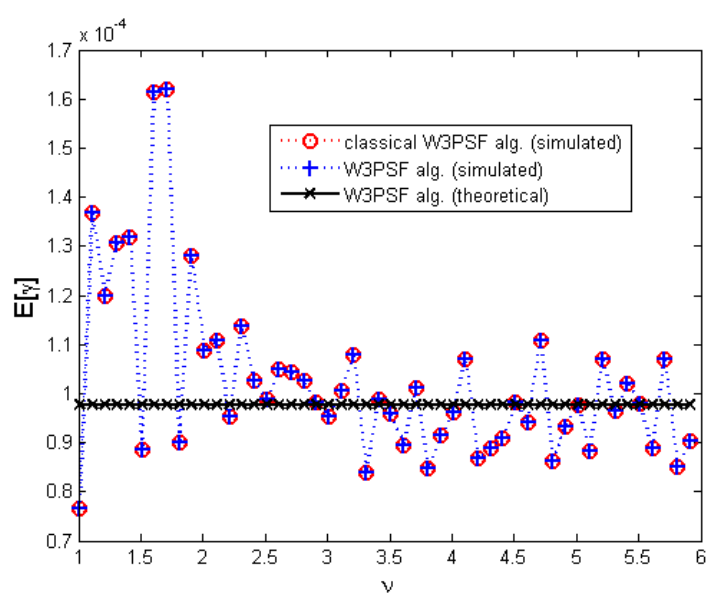

(b)

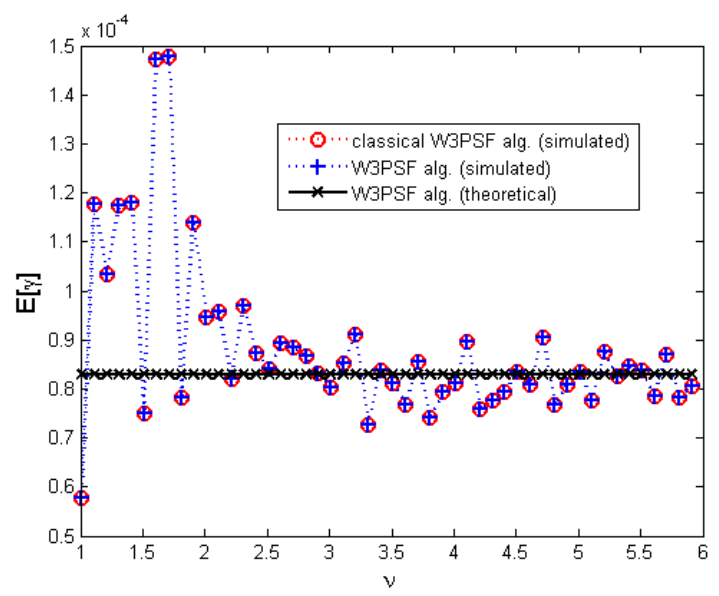

(d)

Fig. 1. Expected sum-squared fitting error obtained by simulations and the proposed analysis versus the number of observed sine-wave cycles $v$ : 3PSF algorithm and equation (28) for $M=17$ (a) and $M=129$ (c); W3PSF algorithm based on the Hann window and equation (27) for $M=17$ (b) and $M=129$ (d). Noisy sinewaves with $S N R=40 \mathrm{~dB}$, sine-wave frequency known a-priori with error $\Delta v=7 \cdot 10^{-3}$ cycles, phase selected at random.

Fig. 1 shows that in the considered cases both the classical and the proposed implementations of the W3PSF algorithm return the same expected sum squared fitting error $E[\gamma]$. This behaviour is obtained for all the considered windows regardless of the $v, M$, and $\Delta v$ values. Also, the obtained $E[\gamma]$ values exhibit larger oscillations when $v$ is small. This is due to a stronger effect on the returned estimates of the image component, which increases as $\Delta v$ increases. Conversely, for high values of $v$ the contribution of the image component becomes negligible with respect to noise and the obtained values of $E[\gamma]$ are very close to the theoretical ones. 
In the following the MSEs of the sine-wave parameter estimators provided by the W3PSF algorithm and the expected sum-squared fitting error are compared with the related values returned by the 3PSF algorithm in order to identify when the W3PSF algorithm can be advantageously applied. Fig. 2 shows the MSEs for the amplitude (Fig. 2(a)), phase (Fig. 2(b)), offset (Fig. 2(c)) and expected sum-squared fitting error (Fig. 2(d)) returned by the 3PSF and the W3PSF algorithms as a function of $\Delta v$ when considering noisy sine-waves characterized by $S N R=30 \mathrm{~dB}$ or $60 \mathrm{~dB}$, respectively, $M=65$ samples, and $v=11.25$ cycles. Both simulation results and values returned by eqs. (18) - (20) and (27) are shown. Also, the related asymptotic CRLB values (21)-(23) are shown in Figs. 2(a)-(c). $\Delta v$ was varied in the range [-0.06, 0.06 ] with a step of $4 \cdot 10^{-3}$ cycles. For each value of $v, 10000$ values of the sine-wave phase were chosen at random in the range $[0,2 \pi)$ rad.

Fig. 2 shows that the previous theoretical analysis well explains the simulation results. Indeed, the number of observed sine-wave cycles and the number of analysed samples $M$ are high enough to ensure that the MSE values returned by simulations are very close to the related theoretical values.

More specifically, Figs. 2(a)-(c) show that the 3PSF algorithm outperforms the W3PSF algorithm when the sine-wave frequency is known with high accuracy, and the range of values of $\Delta v$ in which this happens decreases as $S N R$ increases. This is not the case for the $M S E[\hat{\phi}]$ and $S N R=30 \mathrm{~dB}$, in which the 3PSF algorithm performs better for all considered values of $\Delta v$. This behaviour occurs because the W3PSF algorithm exhibits a higher sensitivity to wideband noise and a lower sensitivity to sine-wave frequency uncertainty. Also, it is worth observing that the $M S E[\hat{\phi}]$ and $M S E[\hat{d}]$ values are almost independent of $\Delta v$, as follows from expressions (19) and (20). When $S N R=30 \mathrm{~dB}$, the $M S E[\hat{A}]$ is almost independent of $\Delta v$ because the contribution to estimation uncertainty of wideband noise prevails over the limited frequency accuracy. Also, the amplitude estimation bias provides a negligible contribution with respect to the related standard deviation. Conversely, when $S N R=60 \mathrm{~dB}$, the $M S E[\hat{A}]$ depends on $\Delta v$ and the related estimation bias is no more negligible. Moreover, MSEs of the phase and offset estimators returned by the 3PSF algorithm exhibit variations due to the effect of the image component, which tends to increase as $\Delta v$ increases. As for the expected sum-squared fitting error, Fig. 2(d) shows that when the contribution of wideband noise prevails, i.e. when the frequency accuracy is high, the 3PSF algorithm outperforms the W3PSF algorithm.

It is also worth observing that when the sine-wave frequency is known with high accuracy (i.e. $\Delta v$ is small so that the contribution of wideband noise dominates the estimation uncertainty) the MSEs of the estimators provided by the 3PSF algorithm are very close to the related CRLBs. Moreover, the range of $\Delta v$ values in which this is true increases as SNR decreases. Also, the phase estimator is quite robust with 
respect to the detrimental effect of image component. Conversely, the MSEs of all estimators returned by the W3PSF algorithm are higher than the related CRLB values, as we expected from the theoretical results.

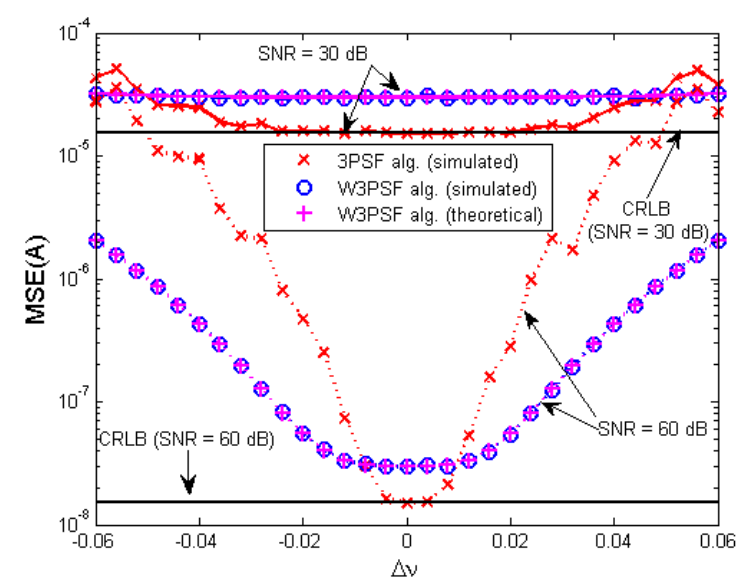

(a)

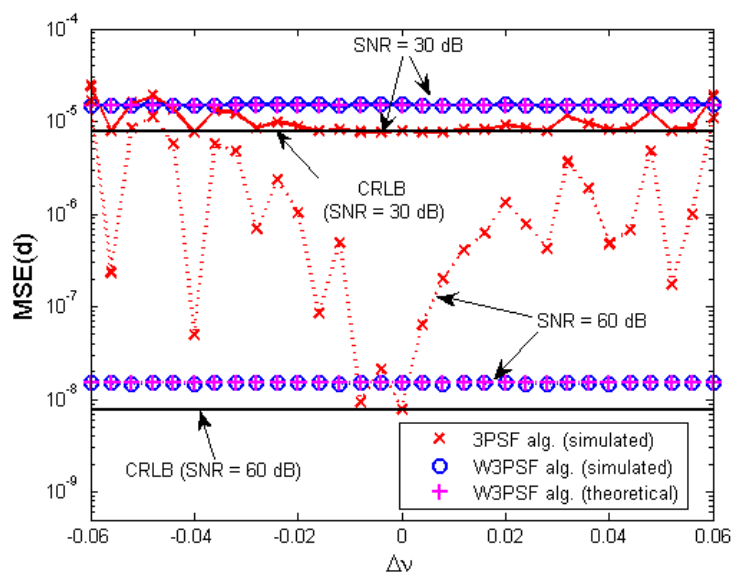

(c)

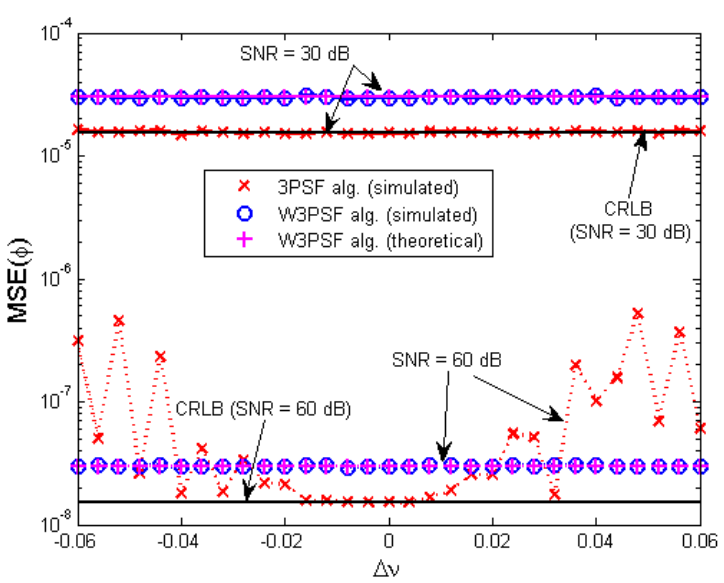

(b)

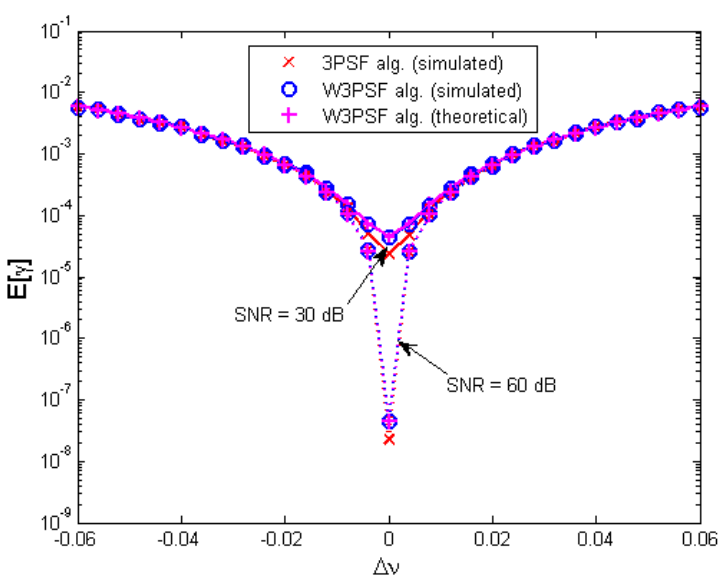

(d)

Fig. 2. Values of $M S E[\hat{A}]$ (a), $M S E[\hat{\phi}]$ (b), $M S E[\hat{d}]$ (c), and $E[\gamma]$ (d) obtained by using the 3PSF algorithm and the W3PSF algorithm based on the Hann window versus $\Delta v$. Both simulation results and values returned by equations (18), (19), (20), and (27) are reported. Also, the related asymptotic CRLB values are shown using a solid line. Noisy sine-waves with $S N R=30 \mathrm{~dB}$ (solid line) or $60 \mathrm{~dB}$ (dotted line), $M=65$ samples, $v=11.25$ cycles, phases selected at random.

Fig. 3 shows the MSEs of the sine-wave parameters returned by the W3PSF algorithm based on the two- and three-term MSD and Minimum Sidelobe Level (MSL) windows as a function of $\Delta v$. The related 
CRLB values are also reported. The simulation parameters are the same as in the previous figure, except that the waveform $S N R$ is $50 \mathrm{~dB}$. It is worth to remember that the $H$-term MSD and MSL windows have the highest sidelobe decay rate (equal to $6(2 H-1) \mathrm{dB} /$ octave) and the smallest maximum sidelobe level, respectively, among the cosine windows with a given number $H$ of coefficients [33]. The $E N B W_{\tilde{w}}$ values of the two- and three-term MSD windows are equal to 1.9444 and 2.6265, respectively, while the values of that parameter for the two- and three-term MSL windows are equal to 1.8223 and 2.3920, respectively.

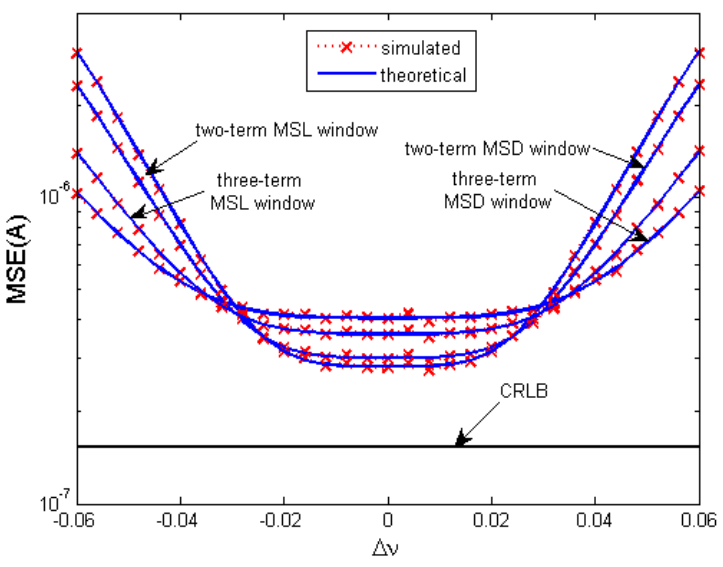

(a)

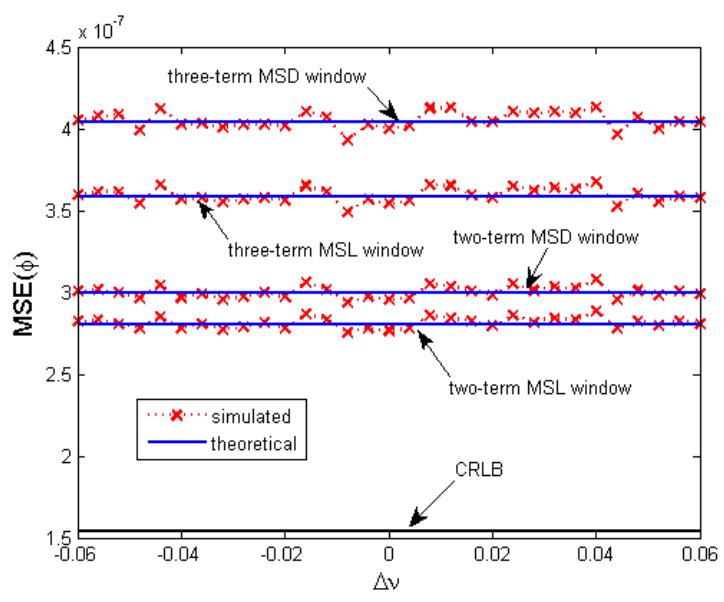

(b)

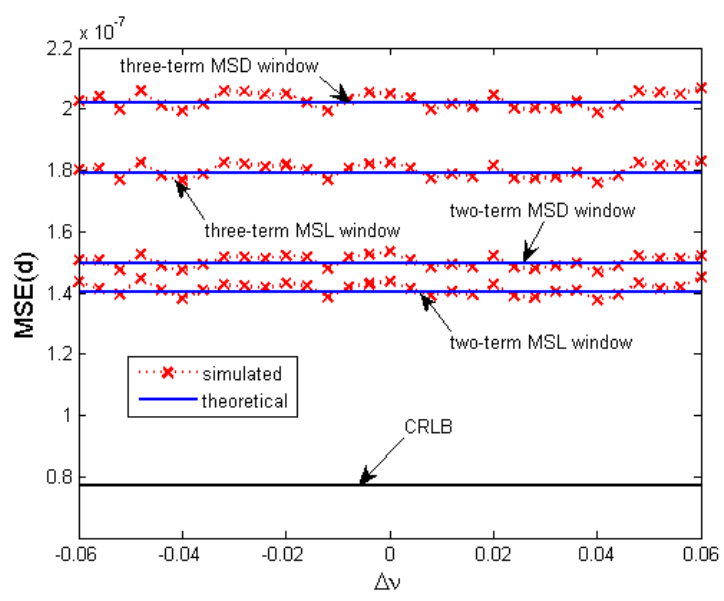

(c)

Fig. 3. Values of $M S E[\hat{A}]$ (a), MSE $[\hat{\phi}]$ (b), MSE $[\hat{d}]$ (c) obtained by using the W3PSF algorithm based on the two- and three-term MSD and MSL windows versus $\Delta v$. Both simulation results and values returned by equations (18), (19), and (20) are reported. Also, the related asymptotic CRLB values are shown using a solid line. Noisy sine-waves with $S N R=50 \mathrm{~dB}, M=65$ samples, $v=11.25$ cycles, phases selected at random. 
Fig. 3 shows that the MSEs returned by simulations are very close to the theoretical ones for all for the considered values of $v$ and $M$. In particular, Fig. 3(a) and (18) show that when $\Delta v$ is small, the contribution of wideband noise dominates. Thus, the amplitude estimation MSE is proportional to $E N B W_{\tilde{w}}$ and the two-term MSL window provides the most accurate results among the considered windows. Conversely, when $\Delta v$ increases the influence of frequency uncertainty prevails on wideband noise and the three-term MSD window provides the best accuracy due to its higher sidelobe decay rate.

Also, Fig. 3, together with (19) and (20), show that the MSEs of the phase and offset estimators are mainly affected by wideband noise and are almost proportional to the $E N B W_{\tilde{w}}$ parameter, so that the best accuracy is achieved when the two-term MSL window is used.

Finally, Fig. 3 quantify the price that must be paid in terms of loss of statistical efficiency (i.e. MSE increase with respect to the CRLB) to reduce the effect of frequency inaccuracy and narrow band disturbances by means of windowing. The effectiveness of windowing in reducing these detrimental effects is analysed in Fig. 4, in which the same simulation parameters as in Fig. 2 are considered, except that the analysed waveforms exhibit a Total Harmonic Distortion THD $=10 \%$ due to a 2nd, a 3rd, and a 4th harmonic with amplitudes in the ratios 4:2:1, respectively. For each value of the frequency $v$ the phases of the fundamental and harmonic components are chosen at random in the range [0,2 $\pi$ ) rad. Again the previous theoretical analysis well explains the simulation results. Figs. 4(a)-(c) show that the 3PSF algorithm outperforms the W3PSF algorithm when the sine-wave frequency is known with high accuracy and $S N R=30 \mathrm{~dB}$, except for the estimated phase for which the 3PSF algorithm performs better for almost all the considered values of $\Delta v$. This behaviour occurs because the W3PSF algorithm exhibits a higher sensitivity to wideband noise and a lower sensitivity to both sine-wave frequency uncertainty and harmonics. Conversely, when SNR $=60 \mathrm{~dB}$ the W3PSF algorithm outperforms the 3PSF algorithm for almost all the considered value of $\Delta v$. Indeed, in this case the contribution to estimation uncertainty of harmonics dominates the effects of both wideband noise and limited frequency accuracy, so that windowing is effective. Also, the MSEs of the phase and offset estimators provided by the 3PSF algorithm exhibit large variations due to the effect of the image component and harmonics, which prevail over that due to wideband noise when $S N R=60 \mathrm{~dB}$.

Fig. 4(d) shows that the 3PSF algorithm slightly outperforms the W3PSF algorithm only when $S N R=30$ $\mathrm{dB}$ and $\Delta v$ is very small, otherwise both algorithms provide almost the same expected sum-squared fitting error. This behaviour occurs because the contribution due to wideband noise dominates the effects of both harmonics and frequency uncertainty only for very small values of $\Delta v$. Conversely, when $S N R=60 \mathrm{~dB}$ and very small $\Delta v$ values are available, the W3PSF algorithm performs better since the contributions of 
both harmonics and frequency uncertainty prevail over the effects of wideband noise, thus making windowing effective.

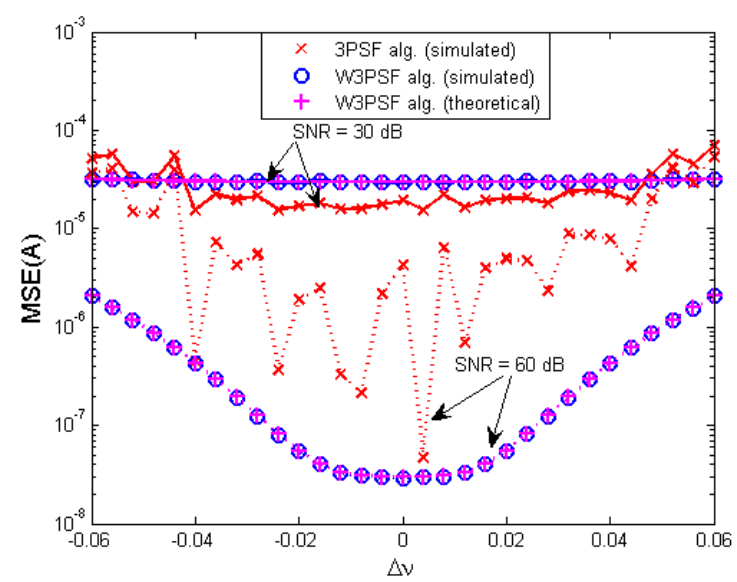

(a)

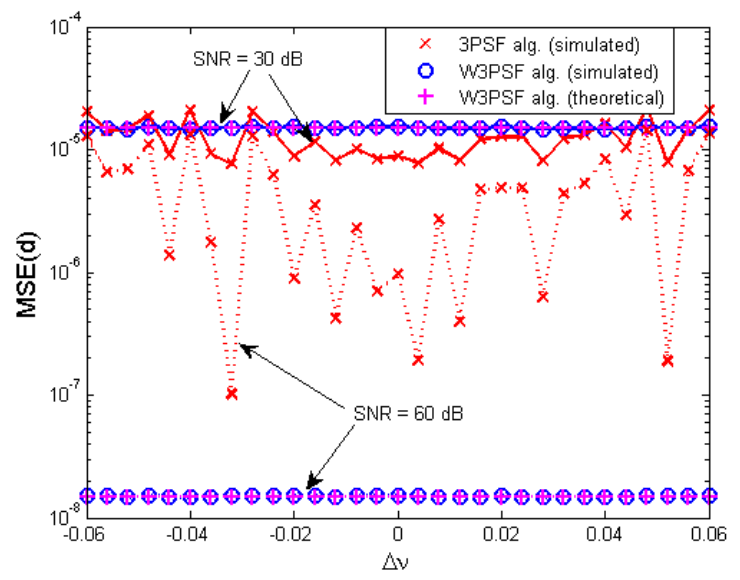

(c)

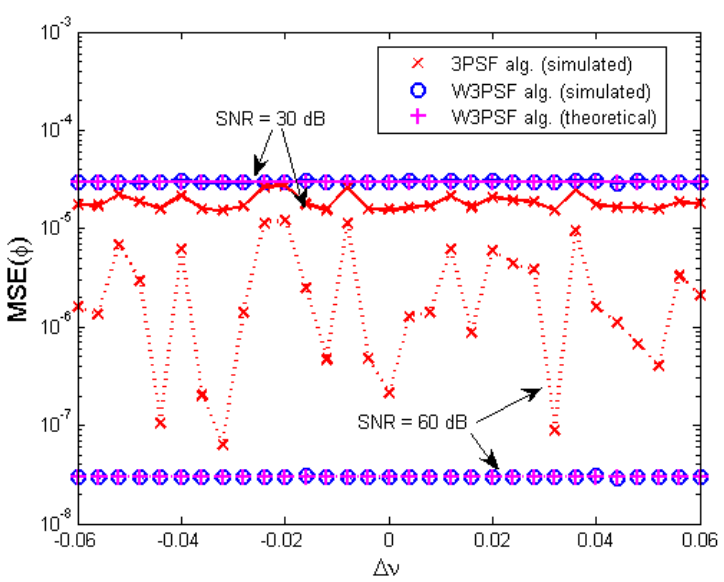

(b)

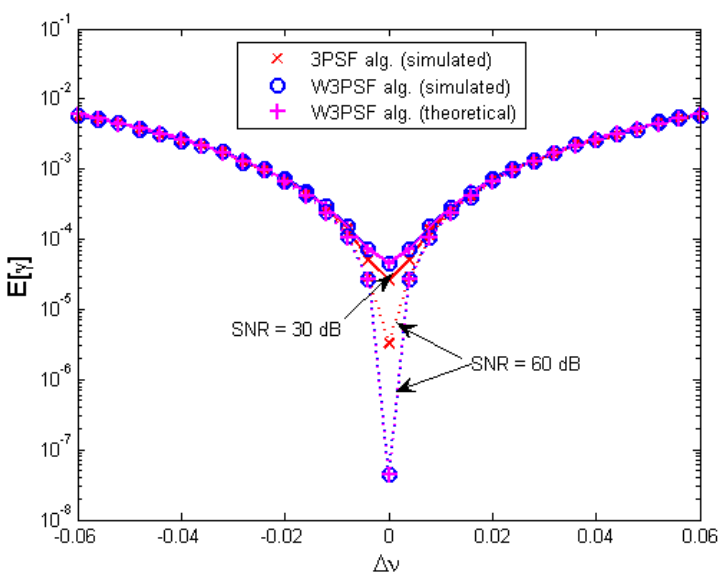

(d)

Fig. 4. Values of $\operatorname{MSE}[\hat{A}]$ (a), $M S E[\hat{\phi}](\mathrm{b}), \operatorname{MSE}[\hat{d}]$ (c), and $E[\gamma]$ (d) obtained using the 3PSF algorithm and the W3PSF algorithm based on the Hann window versus $\Delta v$. Both simulation results and values returned by equations (18), (19), (20), and (27) are reported. Noisy and harmonically distorted sine-waves with $S N R=30 \mathrm{~dB}$ (solid line) or $60 \mathrm{~dB}$ (dotted line), $T H D=10 \% . M=65$ samples, $v=11.25$ cycles, phases selected at random.

To better understand the effect of windowing on distorted sine-waves, the performance of the 3PSF algorithm and the W3PSF based on the Hann window algorithm have been compared when observing a small number of sinewave cycles. Figs. 5 and 6 shows the same accuracy parameters considered in the previous figures as a function of $v$ in the case of noisy (Fig. 5) or noisy and harmonically distorted sinewaves (Fig. 6) when $S N R=30 \mathrm{~dB}$ or $S N R=60 \mathrm{~dB}$, respectively, and $\Delta v=10^{-3}$ cycles. It is worth noticing 
that the contribution of the wideband noise dominates when $S N R=30 \mathrm{~dB}$, i.e. the first term in (27) prevails on the second one. Conversely, when $S N R=60 \mathrm{~dB}$ the effect of the frequency uncertainty overcomes that due to wideband noise. The values of $v$ vary in the range $[1.01,6)$ with a step of 0.1 , while the other simulation parameters are the same as in Fig. 2. The related CRLBs for the amplitude, phase, and offset estimators are also shown in Fig. 5.

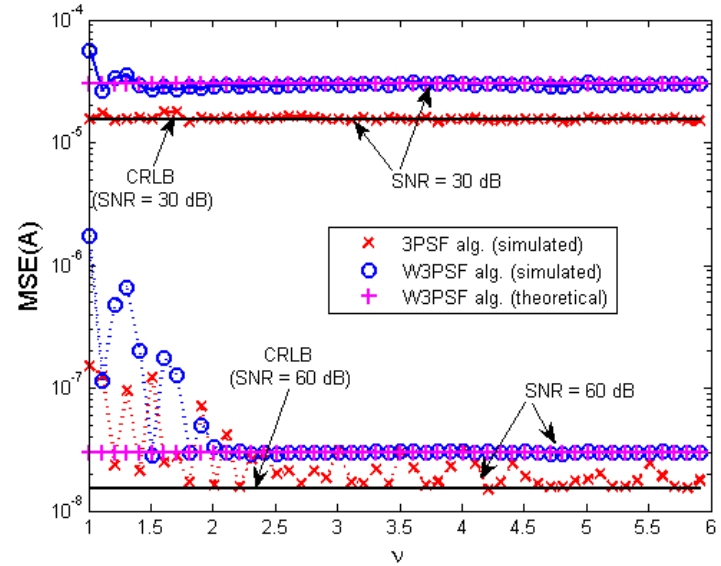

(a)

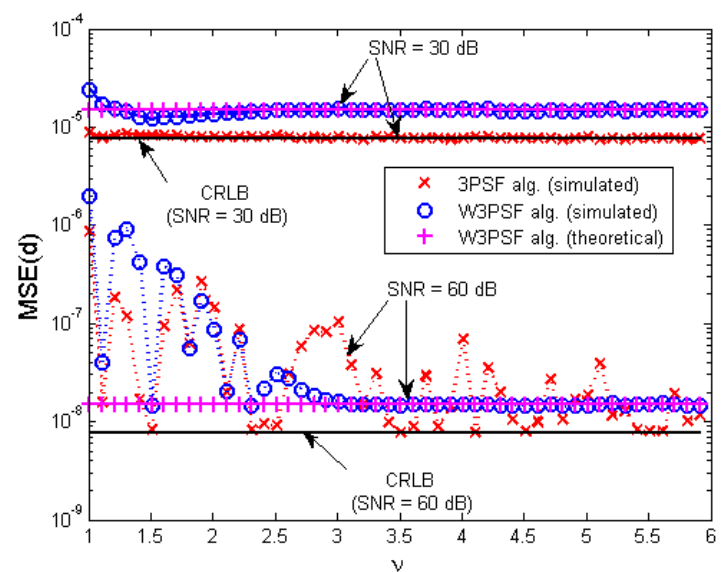

(c)

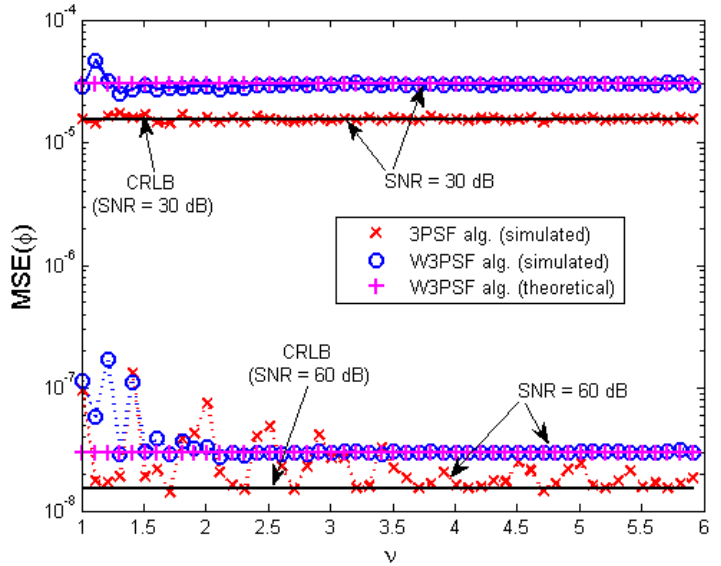

(b)

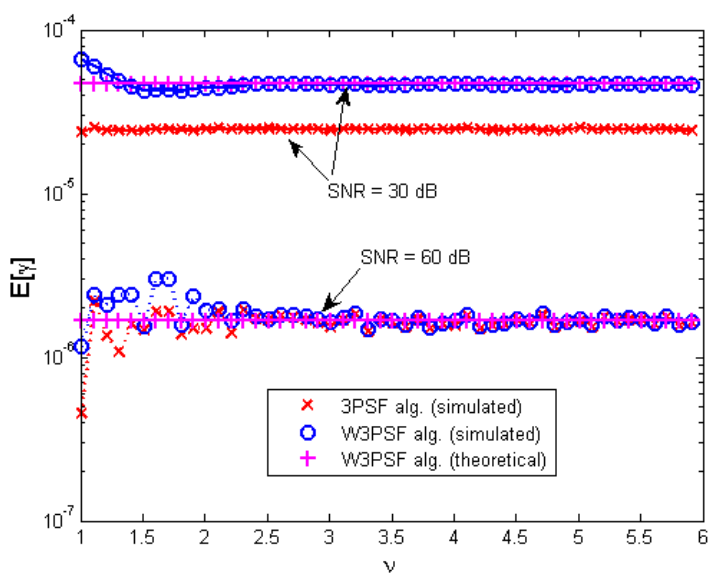

(d)

Fig. 5. Values of $M S E[\hat{A}]$ (a), $M S E[\hat{\phi}]$ (b), $M S E[\hat{d}]$ (c), and $E[\gamma]$ (d) obtained by using the 3PSF algorithm and the W3PSF algorithm based on the Hann window versus the number of observed sine-wave cycles $v$.

Both simulation results and values returned by equations (18), (19), (20), and (27) are reported. Also, the related asymptotic CRLB values are shown in Figs. (a), (b), and (c) using a solid line. Noisy sine-waves with $S N R=30 \mathrm{~dB}$ (solid line) or $60 \mathrm{~dB}$ (dotted line), $\Delta v=10^{-3}$ cycles, $M=65$ samples, phases selected at random. 


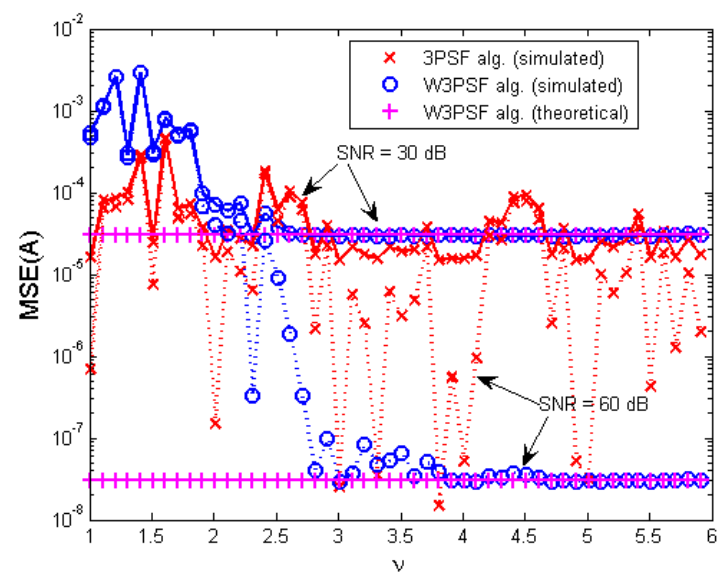

(a)

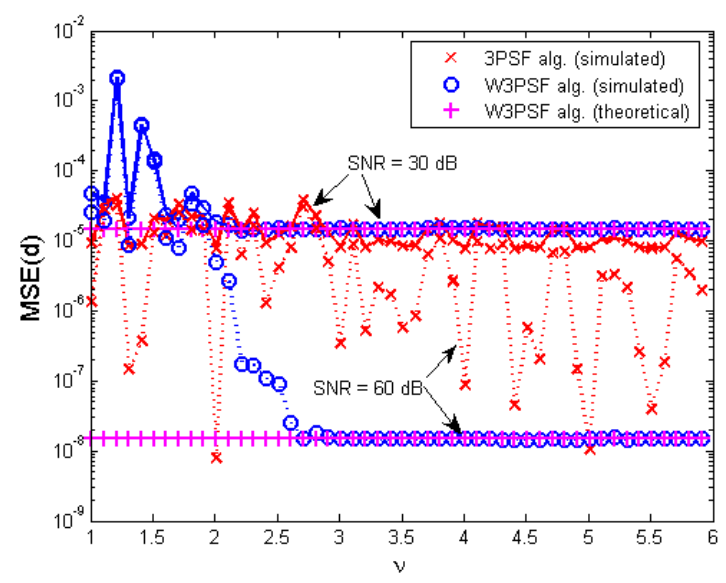

(c)

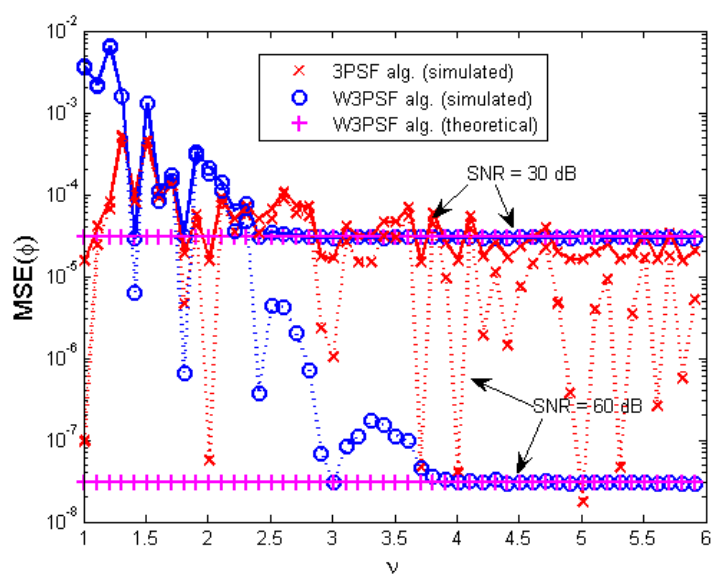

(b)

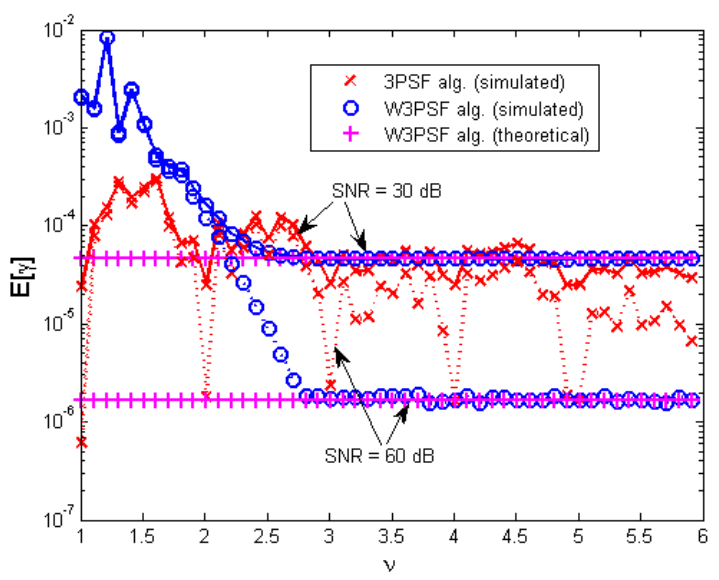

(d)

Fig. 6. Values of $\operatorname{MSE}[\hat{A}]$ (a), $M S E[\hat{\phi}]$ (b), $M S E[\hat{d}]$ (c), and $E[\gamma]$ (d) obtained using the 3PSF algorithm and the W3PSF algorithm based on the Hann window versus the number of observed sine-wave cycles $v$. Both simulation results and values returned by equations (18), (19), (20), and (27) are reported. Noisy and harmonically distorted sine-waves with $S N R=30 \mathrm{~dB}$ (solid line) or $60 \mathrm{~dB}$ (dotted line) and $T H D=10 \% . \Delta v=10^{-3}$ cycles, $M=$

65 samples, phases selected at random.

If noisy sine-waves are of concern, Fig. 5 shows that the 3PSF algorithm outperforms the W3PSF algorithm when $S N R=30 \mathrm{~dB}$ since the effect of wideband noise on the estimation accuracy dominates. Also, the MSEs returned by simulations performed applying the W3PSF algorithm are very close to the respective theoretical values. Conversely, when $S N R=60 \mathrm{~dB}$, the MSEs related to the 3PSF algorithm oscillates because of the effect of the image component, while those related to the W3PSF algorithm are almost equal to the theoretical ones when $v \geq 3$. For the considered values of $\Delta v$ and $M$, the MSEs of the amplitude and phase estimators returned by the 3PSF algorithm are slightly smaller than those achieved by 
the W3PSF algorithm when $v>3.5$. This occurs because of the higher sensitivity to wideband noise due to windowing.

As stated before, wideband noise dominates estimation uncertainty when $S N R=30 \mathrm{~dB}$. Therefore, windowing is useless and the MSEs of the estimators returned by the 3PSF algorithm almost achieve the related CRLBs. Conversely, when $S N R=60 \mathrm{~dB}$, the contribution of wideband noise is comparable with that the effect of the image component and harmonics in most situations so that windowing is advantageous.

Fig. 5(d) shows that the 3PSF algorithm achieves a smaller expected sum-squared fitting error E[ $\gamma]$ than the W3PSF algorithm when $S N R=30 \mathrm{~dB}$. This result agrees with those reported in Figs. 5 (a)-(c). Conversely, both algorithms return almost the same $\mathrm{E}[\gamma]$ value when $S N R=60 \mathrm{~dB}$ and $v>2.5$. Also, the values of $\mathrm{E}[\gamma]$ for the W3PSF algorithm returned by simulations and theoretical analysis are very close to each other for both considered SNR values when $v>2.5$.

It is worth noticing that behaviours very similar to those reported in Fig. 5 have been obtained for the other windows used in Fig. 3.

Noisy and harmonically distorted sine-waves are of concern in Fig. 6. When SNR $=30 \mathrm{~dB}$ the MSEs related to the 3PSF algorithm exhibits large variations, while those related to the W3PSF algorithm are almost constant and very close to the theoretical value for $v>3$. Moreover, the 3PSF algorithm outperforms the W3PSF algorithm when the number of observed cycles $v$ is small. This occurs because the Hann window exhibit a wider spectrum mainlobe than the rectangular window. For the remaining values of $v$ the performances of both algorithms are often very close to each other since the effect of wideband noise is slightly higher than that due to narrow band disturbances. When $S N R=60 \mathrm{~dB}$, the $3 P S F$ often algorithm outperforms the W3PSF algorithm when $1<v<2$, again because of the wider spectrum mainlobe of the Hann window. Conversely, the advantage of windowing is evident when $v>2$. In particular, the MSEs of the amplitude, phase, and offset estimators returned by the W3PSF algorithm are very close to their theoretical values when $v>4, v>4$, and $v>3$, respectively.

As for $E[\gamma]$, the values returned by the 3PSF algorithms exhibit large variations for both values of $S N R$, while those related to the W3PSF algorithm almost coincides with those predicted by theory when $v$ $>3$. In this case the 3PSF and W3PSF algorithms exhibit very close accuracies when $S N R=30 \mathrm{~dB}$, while the W3PSF algorithm largely outperforms the 3PSF algorithm when $S N R=60 \mathrm{~dB}$.

It is worth to observe that by increasing the number of analyzed samples $M$ (e.g. using $M>250$ ) the effect of wideband noise decreases and the W3PSF algorithm outperforms the 3PSF algorithm in most situations also when $S N R=30 \mathrm{~dB}$. 


\section{Conclusions}

Simple expressions for the sine-wave parameter estimators provided by the W3PSF algorithm based on a $H$-term cosine window $(H \geq 1)$ have been proposed in the paper. Specifically, the parameter estimators have been expressed as linear combinations of the values of the weighted DTFT of the analysed waveform evaluated at DC and at the sine-wave frequency. This result is of interest from a practical point of view since it enables low-cost and real-time implementations of the algorithm. Moreover, when the number of analysed waveform cycles is high enough, it has been shown that the derived relationships are well approximated by the estimators returned by the weighted DTFT. Under that constraint, simple expressions providing accurate values for the asymptotic MSEs of the amplitude, phase, and offset estimators returned by the W3PSF algorithm have been proposed. Also, approximated expressions for the asymptotic expected sum-squared fitting error have been obtained. The accuracies of the derived expressions have been verified through computer simulations. Both theoretical and simulation results showed that the classical 3PSF algorithm outperforms the W3PSF algorithm when the contribution to estimation accuracy due to wideband noise prevails over the effects of both the uncertainty of the employed waveform frequency and narrow-band disturbances. Conversely, windowing is advantageous when the influence of limited frequency accuracy and narrow-band disturbances dominate.

\section{Appendix A}

\section{Expressions for the parameter estimators provided by the W3PSF algorithm}

It is worth observing that the weighted sine-wave can be expressed as follows:

$$
W x=\frac{1}{2} W Q P,
$$

where $W$ is the diagonal matrix formed by the samples of the adopted window $w(\cdot)$,

$$
\begin{gathered}
W=\operatorname{diag}[w(-N) w(-N+1) \ldots w(0) \ldots w(N-1) w(N)], \\
Q=\left[\begin{array}{ccc}
e^{-j 2 \pi f N} & e^{j 2 \pi f N} & 1 \\
e^{-j 2 \pi f(N-1)} & e^{j 2 \pi f(N-1)} & 1 \\
& \vdots & \\
e^{j 2 \pi f N} & e^{-j 2 \pi f N} & 1
\end{array}\right],
\end{gathered}
$$

in which $N=(M-1) / 2$, and

$$
P=\left[p p^{*} 2 d\right]^{T},
$$

The least squares solution of (1) can then be expressed as [1]: 


$$
\hat{P}=2\left(Q^{H} W^{H} W Q\right)^{-1} Q^{H} W^{H} W x=2\left(Q_{W}^{H} Q_{W}\right)^{-1} Q_{W}^{H} W x,
$$

where $Q_{W}=W \cdot Q$ is the observation matrix and ${ }^{\mathrm{H}}$ and ${ }^{-1}$ denotes the Hermitian and inverse operators, respectively. The number of acquired signal cycles $\hat{v}$ is assumed to be a-priori known.

After some matrix manipulations we obtain:

$$
Q_{W}^{H} Q_{W}=M \tilde{W}(0)\left[\begin{array}{ccc}
1 & b & a \\
b & 1 & a \\
a & a & 1
\end{array}\right],
$$

where $\tilde{W}(\cdot)$ is the DTFT of the squared window $\tilde{w}(\cdot)$,

$$
a=\frac{\Delta \tilde{W}(-\hat{v})}{\tilde{W}(0)} \text { and } b=\frac{\Delta \tilde{W}(-2 \hat{v})}{\tilde{W}(0)} .
$$

and

$$
Q_{W}^{H} W x=M\left[\begin{array}{c}
X_{\widetilde{w}}(\hat{v}) \\
X_{\tilde{w}}^{*}(\hat{v}) \\
X_{\widetilde{w}}(0)
\end{array}\right],
$$

in which $X_{\widetilde{w}}(\cdot)$ is the DTFT of the windowed signal $x_{\widetilde{w}}(\cdot)$.

The DTFT of the squared window $\tilde{w}(\cdot)$ is defined as [34]:

$$
\tilde{W}(\lambda) \stackrel{\Delta}{=} \sum_{m=0}^{M-1} \tilde{W}(m) e^{-j 2 \pi \lambda\left(m-\frac{M-1}{2}\right) / M},
$$

which for $|\lambda|<<M$ is well approximated by [35]:

$$
\tilde{W}(\lambda) \stackrel{\Delta}{=} \frac{M \sin (\pi \lambda)^{2 H-2}}{\pi} \sum_{h=0}^{2}(-1)^{h} \tilde{c}_{h} \frac{\lambda}{\lambda^{2}-h^{2}},
$$

where $\widetilde{c}_{h}$ are the coefficients of the squared window $\tilde{w}(\cdot)$, given by (11). Observe that $\tilde{W}(\lambda)$ is a realvalued even function.

The DTFT of the windowed signal is defined as:

$$
X_{\tilde{w}}(\lambda)=\sum_{m=0}^{\Delta M-1} X_{\widetilde{w}}(m) e^{-j 2 \pi \lambda\left(m-\frac{M-1}{2}\right) / M},
$$

and, neglecting the contribution of the wideband noise $e(\cdot)$, can be expressed as:

$$
X_{\tilde{w}}(\lambda)=\frac{A}{2 j}\left[\tilde{W}(\lambda-v) e^{j \phi}-\tilde{W}(\lambda+v) e^{-j \phi}\right]
$$

in which the second term represents the contribution of the image component. 
It is worth noticing that $Q_{W}^{H} Q_{W}$ is a symmetric and Hankel matrix. Its inverse, obtained by means of the Symbolic Math Toolbox of MATLAB, is given by:

$$
\left(Q_{W}^{H} Q_{W}\right)^{-1}=\frac{1}{M \tilde{W}(0)}\left[\begin{array}{lll}
\alpha_{11} & \alpha_{12} & \alpha_{13} \\
\alpha_{12} & \alpha_{11} & \alpha_{13} \\
\alpha_{13} & \alpha_{13} & \alpha_{33}
\end{array}\right],
$$

where

$$
\alpha_{11}=\frac{\Delta-a^{2}}{1-2 a^{2}-b^{2}+2 a^{2} b}, \alpha_{12}=\frac{\Delta}{1-2 a^{2}-b^{2}+2 a^{2} b}, \alpha_{13}=-\frac{\Delta}{1+b-2 a^{2}}, \alpha_{33}=\frac{\Delta}{1+b-2 a^{2}} .
$$

By replacing (A.8) and (A.13) into (A.5) it follows:

$$
\left[\begin{array}{l}
\hat{p} \\
\hat{p}^{*} \\
2 \hat{d}
\end{array}\right]=\frac{2}{\widetilde{W}(0)}\left[\begin{array}{lll}
\alpha_{11} & \alpha_{12} & \alpha_{13} \\
\alpha_{12} & \alpha_{11} & \alpha_{13} \\
\alpha_{13} & \alpha_{13} & \alpha_{33}
\end{array}\right]\left[\begin{array}{l}
X_{\tilde{w}}(\hat{v}) \\
X_{\tilde{w}}^{*}(\hat{v}) \\
X_{\tilde{w}}(0)
\end{array}\right] .
$$

from which (5) and (6) are finally obtained.

\section{Appendix B}

\section{Expressions for the asymptotic mean square error of the parameter estimators provided by the}

\section{W3PSF algorithm}

- Asymptotic mean square error of the amplitude estimator

The mean square error of the amplitude estimator is given by:

$$
\operatorname{MSE}[\hat{A}]=\operatorname{bias}^{2}[\hat{A}]+\operatorname{var}[\hat{A}],
$$

where $\operatorname{bias}[\hat{A}]$ and $\operatorname{var}[\hat{A}]$ are the bias and the variance of the estimator $\hat{A}$.

By applying the law of uncertainty propagation [36] to (15) it follows:

$$
\operatorname{var}[\hat{A}] \cong \frac{4}{\widetilde{W}^{2}(0)} \sigma_{X_{\tilde{w}}}^{2},
$$

where $\sigma_{X_{\tilde{w}}}^{2}$ is the variance of the magnitude of the DTFT sample $\left|X_{\tilde{w}}(\hat{v})\right|$, which is given by [37]:

$$
\sigma_{X_{\tilde{w}}}^{2} \cong \frac{M \cdot N N P G_{\tilde{w}}}{2} \sigma^{2}
$$

Since $\tilde{W}(0)=M \widetilde{c}_{0}=M \cdot N P S G_{\tilde{w}}$, using (14) and (B.3), (B.2) becomes:

$$
\operatorname{var}[\hat{A}] \cong \frac{2}{M} E N B W_{\widetilde{w}} \sigma^{2}
$$


As for the estimator bias, from (15) it follows that:

$$
E[\hat{A}] \cong \frac{2}{W(0)} E\left[\left|X_{\tilde{w}}(\hat{v})\right|\right]
$$

where $E[\cdot]$ denotes the expectation operator.

We have [38]:

$$
E\left[\left|X_{\tilde{w}}(\hat{v})\right|^{2}\right] \cong \frac{A^{2}}{4} \tilde{W}^{2}(\Delta v)+M \cdot N N P G_{\tilde{w}} \cdot \sigma^{2},
$$

where $\Delta v=\hat{v}-v$. Since the second term in (B.6) is small when the SNR is high, using the first order Taylor series, $\sqrt{1+x} \cong 1+x / 2$, and (B.2), the following result can be achieved:

$$
E\left[\left|X_{\widetilde{w}}(\hat{v})\right|\right]=\frac{A}{2} \tilde{W}(\Delta v)+\frac{M \cdot N N P G_{\tilde{w}}}{2 A \tilde{W}(\Delta v)} \sigma^{2} .
$$

In practice $\Delta v$ is very small. In this case we have $\sin (\pi \Delta v) \cong \pi \Delta v-\frac{\pi^{3} \Delta^{3} v}{6}$ and $h^{2}-\Delta^{2} v \cong h^{2}$, when $h \geq$ 1. Thus, (A.10) provides:

$$
\tilde{W}(\Delta v) \cong \tilde{W}(0)\left(1-\frac{\pi^{2} \Delta^{2} v}{6}-\frac{\Delta^{2} v}{\tilde{c}_{0}} \sum_{h=1}^{2 H-2} \frac{(-1)^{h} \widetilde{c}_{h}}{h^{2}}\right),
$$

and (B.7) becomes:

$$
\begin{aligned}
E\left[\mid X_{\tilde{w}}(\hat{v})\right] \cong & \frac{A}{2} \tilde{W}(0)\left[1-\left(\frac{\pi^{2}}{6}+\sum_{h=1}^{2 H-2} \frac{(-1)^{h}}{h^{2}} \frac{\tilde{c}_{h}}{\widetilde{c}_{0}}\right) \Delta^{2} v\right] \\
& +\frac{M \cdot N N P G_{\tilde{w}}}{2 A \tilde{W}(0)} \sigma^{2}\left[1+\left(\frac{\pi^{2}}{6}+\sum_{h=1}^{2 H-2} \frac{(-1)^{h}}{h^{2}} \frac{\widetilde{c}_{h}}{\widetilde{c}_{0}}\right) \Delta^{2} v\right] .
\end{aligned}
$$

Since the values of $\sigma^{2}$ and $\Delta^{2} v$ are assumed small, the term containing their product is negligible as compared to the others. Thus, replacing (B.9) in (B.5) and using $\tilde{W}(0)=M \cdot N P S G_{\tilde{w}}$ and (14) we obtain:

$$
E[\hat{A}] \cong A\left[1-\left(\frac{\pi^{2}}{6}+\sum_{h=1}^{2 H-2} \frac{(-1)^{h}}{h^{2}} \frac{\widetilde{c}_{h}}{\widetilde{c}_{0}}\right) \Delta^{2} v+\frac{E N B W_{\tilde{w}}}{2 M \cdot S N R}\right],
$$

where $S N R=A^{2} /\left(2 \sigma^{2}\right)$ is the Signal-to-Noise Ratio of the analysed waveform. The last term in (B.10) represents the contribution of wideband noise which becomes negligible with respect to the contribution due to a-priori known waveform frequency $\hat{v}$ when a sufficiently high number of samples $M$ are considered. Thus, from (B.10) it easily follows that the amplitude estimator bias is given by:

$$
\operatorname{bias}[\hat{A}] \cong A\left(\frac{\pi^{2}}{6}+\sum_{h=1}^{2 H-2} \frac{(-1)^{h}}{h^{2}} \frac{\widetilde{c}_{h}}{\widetilde{c}_{0}}\right) \Delta^{2} v .
$$

Thus, using (B.4) and (B.11) the expression (18) can be finally derived. 


\section{- Asymptotic mean square error of the phase estimator}

Since $\tilde{W}(\lambda)$ is a real-valued function, the estimator $\hat{\phi}$ given by (16) does not depend on $\hat{v}$. In addition, computer simulations showed that for noisy sine-waves we have $\operatorname{bias}[\hat{\phi}]<<s t d[\hat{\phi}]$, so that:

$$
\operatorname{MSE}[\hat{\phi}] \cong \operatorname{var}[\hat{\phi}] .
$$

From (16) the phase estimator variance due to wideband noise is given by [37]:

$$
\operatorname{var}[\hat{\phi}] \cong \frac{M \cdot N N P G_{\tilde{w}}}{2\left|X_{\tilde{w}}(\hat{v})\right|^{2}} \sigma^{2}
$$

Assuming $\Delta v$ negligible, since $\tilde{W}(0)=M \cdot \widetilde{c}_{0}=M \cdot N P S G_{\tilde{w}}$, by using (14) and (15) we obtain:

$$
\operatorname{var}[\hat{\phi}] \cong \frac{1}{M \cdot S N R} E N B W_{\widetilde{w}},
$$

By replacing (B.14) in (B.12) the expression (19) is then derived.

\section{- Asymptotic mean square error of the offset estimator}

From (17) it follows that the offset estimator does not depend on the waveform frequency $\hat{v}$. Thus, it follows that the related mean square error is due only to wideband noise. Using a derivation similar to the one used above to analyse the influence of noise on amplitude estimator, it results that the bias of the offset estimator is half that of the amplitude estimator, i.e.:

$$
\operatorname{bias}[\hat{d}] \cong \frac{E N B W_{\tilde{w}}}{2 A \cdot M} \sigma^{2} \text {. }
$$

Similarly, from (17) and since the variance of $\left|X_{\tilde{w}}(0)\right|$ is twice the value returned by (B.3), we obtain:

$$
\operatorname{var}[\hat{d}] \cong \frac{1}{M} E N B W_{\widetilde{w}} \sigma^{2} .
$$

By comparing (B.15) with (B.16) and remembering that we are analyzing a high number of waveform samples $M$, we have bias $[\hat{d}]<<s t d[\hat{d}]$. Thus:

$$
\operatorname{MSE}[\hat{d}] \cong \operatorname{var}[\hat{d}] .
$$

Finally, by replacing (B.16) in (B.17) the expression (20) is obtained. 


\section{Appendix C}

\section{Expression for the asymptotic expected sum-squared fitting error returned by the W3PSF algorithm}

Since the sine-wave phase $\phi$ is referred to the centre of the observation interval, when a large number of samples are analysed and the number of waveform cycles is high enough, the sum-squared fitting error is given by [8]:

$$
\gamma=\frac{1}{M} \sum_{m=0}^{M-1} \varepsilon^{2}(m) \cong \Delta^{2} d+\frac{\Delta^{2} A}{2}+\frac{A^{2} \Delta^{2} \phi}{2}+\frac{\pi^{2} A^{2} \Delta^{2} v}{6}
$$

where $\hat{A}, \hat{\phi}$, and $\hat{d}$ are the amplitude, phase, and offset parameters estimated by the SW3PSF algorithm, $\Delta A=\hat{A}-A, \Delta \phi=\hat{\phi}-\phi$, and $\Delta d=\hat{d}-d$.

By taking the expectation of (C.1) we have:

$$
E[\gamma] \cong \operatorname{MSE}[\hat{d}]+\frac{1}{2} \operatorname{MSE}[\hat{A}]+\frac{A^{2}}{2} \operatorname{MSE}[\hat{\phi}]+\frac{\pi^{2} A^{2}}{6} \operatorname{MSE}[\hat{v}] .
$$

By replacing (18) - (20) in (C.2) we obtain:

$$
E[\gamma] \cong \frac{3 E N B W_{\tilde{w}}}{M} \sigma^{2}+\frac{\pi^{2} A^{2}}{6} \operatorname{MSE}(\hat{v})+\frac{A^{2}}{2}\left(\frac{\pi^{2}}{6}+\sum_{h=1}^{2 H-1} \frac{(-1)^{h}}{h^{2}} \frac{\widetilde{c}_{h}}{\widetilde{c}_{0}}\right)^{2} \Delta_{\hat{v}}^{4} .
$$

Observe that $\Delta^{2} v$ is assumed to be small. Thus, the last term in (C.3) can be neglected as compared with the second term and expression (27) is finally obtained.

\section{References}

[1] S.M. Kay, Fundamentals of Statistical Signal Processing: Estimation Theory (Prentice-Hall, Upper Saddle River, NJ, 1993).

[2] T. Söderström, P. Stoica, System Identification (Englewood Cliffs, Prentice-Hall, N. J, 1989).

[3] IEEE Standard for Digitizing Waveform Recorders, IEEE Std. 1057-2007, 2007.

[4] IEEE Std. 1241, Standard for Terminology and Test Methods for Analog-to-Digital Converters, Dec., 2010.

[5] European Project DYNAD. Methods and Draft Standards for the Dynamic Characterization and Testing of Analog-to-Digital Converters.

[6] P. Händel, Properties of the IEEE-STD-1057 four-parameter sine wave fit algorithm, IEEE Trans. Instrum. Meas. 49 (6) (2000) 1189-1193.

[7] T. Andersson, P. Händel, IEEE standard 1057, Cramér-Rao bound and the parsimony principle, IEEE Trans. Instrum. Meas. 55(1) (2006) 44-53.

[8] D. Belega, D. Petri, D. Dallet, Noise power estimation by the three-parameter and four-parameter sine-fit algorithms, IEEE Trans. Instrum. Meas. 61(12) (2012) 3234-3240. 
[9] F.C. Alegria, Bias of amplitude estimation using three-parameter sine fitting in the presence of additive noise, Meas. 42 (2009) 748-756.

[10] P. Händel, Amplitude estimation using IEEE-std-1057 three-parameter sine wave fit: statistical distribution, bias and variance, Meas. 43 (2010) 766-770.

[11] T.Z. Bilau, T. Megyeri, A. Sárhegyi, J. Márkus, I. Kollár, Four-parameter fitting of sine wave testing result: Iteration and convergence, Comput. Stand. Interfaces 26(1) (2003) 51-56.

[12] M. Fonseca da Silva, P.M. Ramos, A. Cruz Serra, A new four parameter sine fitting procedure, Meas. 35(2) (2004) 131-137.

[13] K.-F. Chen, Y.-M. Xue, "Improving four-parameter sine wave fitting by normalization,” Comput. Stand. Interfaces, 29(2) (2007) 184-190.

[14] A. Moschitta, P. Carbone, "Statistical efficiency of sinewave fitting when using nonlinear quantizers," in Proc. IEEE Instrum. Meas. Technol. Conf., Ottawa, ON, Canada, May 2005, pp. 510-515.

[15] S. Negusse, P. Händel, P. Zetterberg, IEEE-std-1057 three parameter sine wave fit for SNR estimation: performance analysis and alternative estimators, IEEE Trans. Instrum. Meas. 63(6) (2014) 1514-1523.

[16] D.C. Rife, G.A. Vincent, Use of the discrete Fourier transform in the measurement of frequencies and levels of tones, Bell Syst. Tech. J. 49 (1970) 197-228.

[17] C. Offelli, D. Petri, The influence of windowing on the accuracy of multifrequency signal parameter estimation, IEEE Trans. Instrum. Meas. 41 (2) (1992) 256-261.

[18] D. Belega, D. Petri, Sine-wave parameter estimation by interpolated DFT method based on the new cosine windows with high interference rejection capability, Digit. Signal Process. 33 (2014) 60-70.

[19] B.G. Quinn, Estimation of frequency, amplitude, and phase from the DFT of a time series, IEEE Trans. Signal Process. 45 (1997) 814-817.

[20] B.G. Quinn, Frequency estimation using tapered data, Proc. ICASSP 3 (2006) III-73-III-76.

[21] B.G. Quinn, Recent advances in rapid frequency estimation, Digital Signal Process. 19 (6) (2008) 942-948.

[22] Z-G Chen, K.H. Wu, R. Dahlhaus, Hidden frequency estimation with data tapers, J. Time Ser. Anal., 21 (2) (2000) 113-142.

[23] E. Aboutanios, B. Mulgrew, Iterative frequency estimation by interpolation on Fourier coefficients, IEEE Trans. Signal Process. 53 (4) (2005) 1237-1241.

[24] S. Ye, E. Aboutanios, An algorithm for the parameter estimation of multiple superimposed exponentials in noise, Proc. ICASSP (2015) 3457-3461.

[25] C. Candan, A method for fine resolution frequency estimation from three DFT samples, IEEE Signal Process. Lett. 18 (6) (2011) 351-354.

[26] Y. Liu, Z. Nie, Z. Zhao, Q.H. Liu, Generalization of iterative Fourier interpolation algorithm for single frequency estimation, Digital Signal Process. 21 (1) (2011) 141-149.

[27] C. Candan, Fine resolution frequency estimation from three DFT samples: case of windowed data, Signal Process. 114 (2015) 245-250. 
[28] H. Wen, S. Guo, Z. Teng, Y. Yang, Frequency estimation of distorted and noisy signals in power systems by FFT-based approach, IEEE Trans. Power Syst. 29(2) (2014) 765-774.

[29] P. Carbone, E. Nunzi, D. Petri, Frequency-domain-based least-squares estimation of multifrequency signal parameters, IEEE Trans. Instrum. Meas. 49(2) (2000) 555-558.

[30] I. Djurović, V. Katkovnik, L.J. Stanković, Median filter based realizations of the robust time-frequency distibutions, Signal Process. 81 (7) (2001) 1771-1776.

[31] I. Djurović, V.V. Lukin, Estimation of single-tone signal frequency by using the L-DFT, Signal Process. 87 (6) (2007) 1537-1544.

[32] F.J. Harris, On the use of windows for harmonic analysis with the discrete Fourier transform, Proceedings of the IEEE 66 (1) (1978) 51-83.

[33] A.H. Nuttall, Some windows with very good sidelobe behavior, IEEE Trans. Acoust. Speech Signal Process. ASSP-29 (1) (1981) 84-91.

[34] S. Gori, C. Narduzzi, Application of a phase measurement algorithm to digitizing oscilloscope characterization, IEEE Trans. Instrum. Meas. 49 (6) (2000) 1211- 1215.

[35] D. Belega, D. Petri, Accuracy analysis of the multicycle synchrophasor estimator provided by the interpolated DFT algorithm, IEEE Trans. Instrum. Meas. 62(5) (2013) 942-953.

[36] Guide for the expression of uncertainty in measurement (GUM), International Organization for Standardization, Switzerland, second edition, 1995.

[37] M. Novotný, D. Slepička, M. Sedláček, Uncertainty analysis of the RMS value and phase in frequency domain by noncoherent sampling, IEEE Trans. Instrum. Meas. 56(3) (2007) 983-989.

[38] D. Petri, Frequency-domain testing of waveform digitizers, IEEE Trans. Instrum. Meas. 51(3) (2002) 445-453. 